\title{
Energy Fluxes in Coastal Trapped Waves
}

\author{
R. C. Musgrave \\ Woods Hole Oceanographic Institution, Woods Hole, Massachusetts
}

(Manuscript received 20 August 2018, in final form 10 September 2019)

\begin{abstract}
The calculation of energy flux in coastal trapped wave modes is reviewed in the context of tidal energy pathways near the coast. The significant barotropic pressures and currents associated with coastal trapped wave modes mean that large errors in estimating the wave flux are incurred if only the baroclinic component is considered. A specific example is given showing that baroclinic flux constitutes only $10 \%$ of the flux in a mode- 1 wave for a reasonable choice of stratification and bathymetry. The interpretation of baroclinic energy flux and barotropic-to-baroclinic conversion at the coast is discussed: in contrast to the open ocean, estimates of baroclinic energy flux do not represent a wave energy flux; neither does conversion represent the scattering of energy from the tidal Kelvin wave to higher modes.
\end{abstract}

\section{Introduction}

Coastal trapped waves form a distinct class of wave motions in the ocean, relying on the presence of a topographic waveguide for their propagation. Unlike freely propagating inertia-gravity waves, there are no lower frequency limits for coastal trapped waves, which makes them an important mechanism for the transfer of subinertial energy along coastlines. They are often wind driven (e.g., Clarke 1977), but at high latitudes can be tidally driven (e.g., Cartwright 1969), and in the latter case can mediate the transfer of tidal energy toward dissipation in the ocean through scattering to smaller scales.

In an effort to quantify ocean mixing by the tides, significant work has focused on the energy budget of the internal tide, whose dissipation is thought to be important for deep diapycnal transformations. Here, the term "internal tide" refers to waves at tidal frequencies that rely on stratification and thus include vertical current shears. Throughout much of the ocean the internal tide is superinertial, forming a freely propagating internal wave that can transit ocean basins. The dynamics of superinertial internal tides in a flat bottom ocean are well described by vertical modes, the gravest of which is, to leading order, barotropic, while the higher modes that constitute the internal tide are baroclinic. Baroclinic motions have zero depth mean currents and pressure,

Corresponding author: R. C. Musgrave,rmusgrave@whoi.edu and are associated with isopycnal displacements and shears in midwater column (Salmon 1998). Thus, for these flat bottom modes, the astronomically forced gravest wave mode is easily separated simply by subtracting the depth mean currents, isolating the baroclinic internal tides [for a discussion of some of the assumptions in this separation, see Kelly (2016)]. Internal tide energy budgets can then be constructed, quantifying the conversion of energy from the barotropic to baroclinic modes at topography (Nash et al. 2005; Kang and Fringer 2012), a key energy transfer describing the scattering of wave energy from large, astronomically forced scales toward smaller scales at which dissipation and mixing occur.

At high latitudes (poleward of $30^{\circ}$ for diurnals and $74^{\circ}$ for semidiurnals), however, subinertial internal tides are topographically trapped, forming coastally trapped waves at the coasts. The structure of these waves is very different to that of freely propagating superinertial internal waves: not only do they couple barotropic and baroclinic motions, they also have nonseparable structure in the cross-shore and vertical directions. Both subinertial coastal trapped waves and their superinertial analogs [the so-called "leaky" coastal trapped waves discussed by Dale and Sherwin (1996)] have modal structures with large depth means, meaning that they cannot be isolated by subtracting a barotropic mode. Even the gravest mode "barotropic" Kelvin wave is not truly barotropic over coastal bathymetry, as it includes small baroclinic components at the shelfbreak. As such, 
the conversion of barotropic to baroclinic energy no longer describes the scattering of wave energy from the gravest to higher modes, instead quantifying the exchange of energy between motions that do not constitute free wave modes on the coast. Under some circumstances it may still be possible to track energy exchange between the gravest and higher coastal modes, however this quantity is of uncertain importance in a coastal context, as it relies on the underlying assumption that only the gravest mode is directly forced by the astronomical potential. In coastal regions this is questionable, as all wave modes have a barotropic component onto which the depth uniform astronomical tide generating force could project.

Nevertheless, recent work has sought to examine the energetics of these trapped waves as an important component of ocean processes near topography and at the basin boundaries (Tanaka et al. 2010; Niwa and Hibiya 2011; Müller 2013; Tanaka et al. 2013; Fer et al. 2015; Klymak et al. 2016; Musgrave et al. 2017; Li et al. 2017; Masunaga et al. 2017; Hughes and Klymak 2019). Many authors (including this author) have calculated energy fluxes or constructed energy budgets for subinertial trapped internal tides by undertaking the same analysis that has been successfully applied to superinertial tides over a flat bottom (e.g., Kang and Fringer 2012). The purpose of this short contribution is to point out that the interpretation of baroclinic energy flux and barotropic-baroclinic conversion is very different near the coast than in the open ocean. The assumption that baroclinic energy flux represents a wave flux leads to large errors when trapped waves may be present, as it essentially decomposes the observed currents and pressures into structures that do not resemble the free waves on the coast. The baroclinic tide, identified in this manner, does not constitute a wave mode on the coast, and flux estimates are an underestimate of the true wave energy in the modes carrying the baroclinic signal as the depth mean component of those modes has been incorrectly attributed to the gravest wave mode and removed.

In coastal regions, energy pathways are dictated by the efficiency of astronomical forcing on each of the modes and their subsequent scattering of energy amongst themselves. However, tracking wave energy at the ocean's boundaries is significantly more complicated than in the interior for both practical and conceptual reasons. Not only is it more difficult in practice to isolate individual coastal trapped modes, they are not, in general, orthogonal, making interpretations of individual wave flux of uncertain utility.

Some basic physics for subinertial coastal trapped waves is reviewed in section 2 , highlighting important features of their behavior. An example of a wave flux calculation is given in section 3, illustrating the significant errors associated with a vertical mode decomposition. Section 4 is a summary.

\section{A review of coastal trapped waves and their energetics}

We consider a coast at $x=0$ such that $x$ is directed in the offshore direction and $y$ is alongshore. Following Wang and Mooers (1976), we outline the derivation and governing equations for stratified, subinertial, linear coastal trapped waves over a sloping bottom. Solutions are assumed to be wavelike and propagate alongshore, thus being of the form

$$
(u, v, w, \rho, p)=\Re\left\{\left(\tilde{u}, \tilde{v}, \tilde{w}, \tilde{\rho}^{\prime}, \tilde{p}\right)(x, z) e^{[i(l y+\omega t)]}\right\},
$$

where $u, v$, and $w$ are velocity components, $\rho^{\prime}$ is the density perturbation from a background profile, $p$ is the pressure, $\omega$ is the wave frequency, and $l$ is the along-shelf wavelength. The linearized equations of motion are

$$
\begin{aligned}
i \omega \tilde{u}-f \tilde{v} & =-\frac{1}{\rho_{0}} \frac{\partial \tilde{p}}{\partial x}, \\
i \omega \tilde{v}+f \tilde{u} & =-\frac{1}{\rho_{0}} i l \tilde{p}, \\
\frac{\partial \tilde{p}}{\partial z} & =-g \tilde{\rho}^{\prime}, \\
\frac{\partial \tilde{u}}{\partial x}+i l \tilde{v}+\frac{\partial \tilde{w}}{\partial z} & =0, \quad \text { and } \\
i \omega \tilde{\rho}^{\prime}-\frac{\rho_{0} N^{2}}{g} \tilde{w} & =0 .
\end{aligned}
$$

The equations can be combined to form a single expression in pressure,

$$
\frac{\partial^{2} \tilde{p}}{\partial x^{2}}+\left(f^{2}-\omega^{2}\right) \frac{\partial}{\partial z} \frac{1}{N^{2}} \frac{\partial \tilde{p}}{\partial z}-l^{2} \tilde{p}=0,
$$

which is solved subject to the no-normal-flow boundary condition at the bottom:

$$
\frac{1}{N^{2}} \frac{\partial \tilde{p}}{\partial z}=-\frac{H_{x}}{f^{2}-\omega^{2}}\left(\frac{\partial \tilde{p}}{\partial x}+\frac{l f}{\omega} \tilde{p}\right) \quad \text { at } \quad z=-H(x)
$$

a linearized free surface:

$$
\tilde{w}=i \omega \frac{\tilde{p}}{g \rho_{0}} \quad \text { at } \quad z=0,
$$


no flow through the coastal boundary:

$$
\frac{\partial \tilde{p}}{\partial x}=-\frac{f l}{\omega} \tilde{p} \quad \text { at } \quad x=0,
$$

and a decaying signal far offshore:

$$
\tilde{p} \rightarrow 0 \quad \text { at } \quad x \rightarrow \infty .
$$

Solutions to Eq. (6) are nonnormal, two-dimensional modes, whose structure is typically sought numerically by resonant iteration, though analytic expressions have been derived for some specific topographies and stratifications (Huthnance 1978). Once the pressure mode is known, the remaining variables are defined by the following polarization relations:

$$
\begin{aligned}
& \tilde{u}=-i \frac{\left(f l \tilde{p}+\omega \partial_{x} \tilde{p}\right)}{\rho_{0}\left(f^{2}-\omega^{2}\right)}, \\
& \tilde{v}=\frac{\left(f \partial_{x} \tilde{p}+\omega l \tilde{p}\right)}{\rho_{0}\left(f^{2}-\omega^{2}\right)}, \\
& \tilde{w}=-\frac{i \omega}{\rho_{0} N^{2}} \partial_{z} \tilde{p}, \quad \text { and } \\
& \tilde{\rho}^{\prime}=-\frac{\partial_{z} \tilde{p}}{g} .
\end{aligned}
$$

An energy equation can be formed from Eqs. (1)-(5), and the time rate of change of the total energy is given by the divergence of the wave flux $\mathbf{u} p$ :

$$
\frac{\partial E}{\partial t}=-\nabla \cdot(\mathbf{u} p) .
$$

It is apparent by inspection of Eqs. (11)-(14) that all of the energy flux in these individual wave modes is alongshore, because $v$ and $p$ are in phase, whereas $u-p$ and $w-p$ are in quadrature. An expression for the alongshore flux can be derived by setting $\tilde{p} \in \Re$; then

$$
\begin{aligned}
& p=\tilde{p} \cos (l y+\omega t) \quad \text { and } \\
& v=\frac{\left(f \partial_{x} \tilde{p}+\omega l \tilde{p}\right)}{\rho_{0}\left(f^{2}-\omega^{2}\right)} \cos (l y+\omega t),
\end{aligned}
$$

and therefore the depth and cross-shelf structure of along-shelf flux for a single mode is

$$
\langle v p\rangle=\frac{1}{T} \int_{0}^{T} v p d t=\frac{1}{2} \tilde{p} \frac{\left(f \partial_{x} \tilde{p}+\omega l \tilde{p}\right)}{\rho_{0}\left(f^{2}-\omega^{2}\right)},
$$

where $T$ is the period of the wave. The total along-shelf flux is calculated by integrating in depth and offshore distance:

$$
\mathscr{F}=\int_{0}^{\infty} \int_{-H}^{0} \frac{\tilde{p} \tilde{v}}{2} d z d x
$$

Notice that, unlike freely propagating inertia-gravity waves for which the depth-integrated flux for a single plane wave is horizontally uniform, the sign of the depth-integrated along shelf flux in coastal trapped waves changes with position across the shelf. The number of zero crossings is determined by the product of the pressure and alongshore velocity component, which each have the same number of cross-shore nodes (though at different positions) as the mode number. As a result, the number of zero crossings in flux is $2 n$, where $n$ is the mode number (Crawford 1984). It is the cross-shelf and depth integral of the flux has a sign that is consistent with the gradient of the dispersion curve, that is, the group velocity of the wave.

Solutions to Eq. (6) do not, in general, exhibit the convenient property of orthogonality associated with flat bottom modes, with implications for wave energy as discussed below. However, in the long wave limit $(\omega \ll f$, and $L_{y} \gg L_{x}$, with $L_{y}$ being the along-shelf scale and $L_{x}$ being the cross-shelf scale) the alongshore flow becomes geostrophically balanced and the governing equation becomes

$$
\frac{\partial^{2} \tilde{p}}{\partial x^{2}}+f^{2} \frac{\partial}{\partial z} \frac{1}{N^{2}} \frac{\partial \tilde{p}}{\partial z}=0
$$

with an accompanying orthogonality relation for mode numbers $n$ and $m$ :

$$
\delta_{n m} D_{n}=\frac{1}{f}\left(\left.\int_{-h}^{0} p_{n} p_{m} d z\right|_{x=0}+\left.\int_{0}^{\infty} \frac{d H}{d x} p_{n} p_{m} d x\right|_{z=-h}\right) .
$$

The normalization $D_{n}$ must be chosen with care to ensure the conservation of energy in each mode along a slowly varying coast (Brink 1989). That short coastal trapped waves are not orthogonal presents difficulties in understanding the partition of wave energy amongst individual modes when more than one mode is present. As an example, consider the along-shelf flux of two coastal trapped wave modes at the same frequency

$$
\langle v p\rangle=\left\langle v_{1} p_{1}\right\rangle+\left\langle v_{2} p_{2}\right\rangle+\left\langle v_{1} p_{2}\right\rangle+\left\langle v_{2} p_{1}\right\rangle,
$$

where angle brackets indicate time averaging. Using Eq. (16), the cross terms have the form

$$
\begin{aligned}
\left\langle v_{1} p_{2}\right\rangle & =\left\langle\tilde{v}_{1} \tilde{p}_{2} \cos \left(l_{1} y-\omega t\right) \cos \left(l_{2} y-\omega t\right)\right\rangle \\
& =\frac{1}{2} \tilde{v}_{1} \tilde{p}_{2} \cos \left[\left(l_{1}-l_{2}\right) y\right],
\end{aligned}
$$



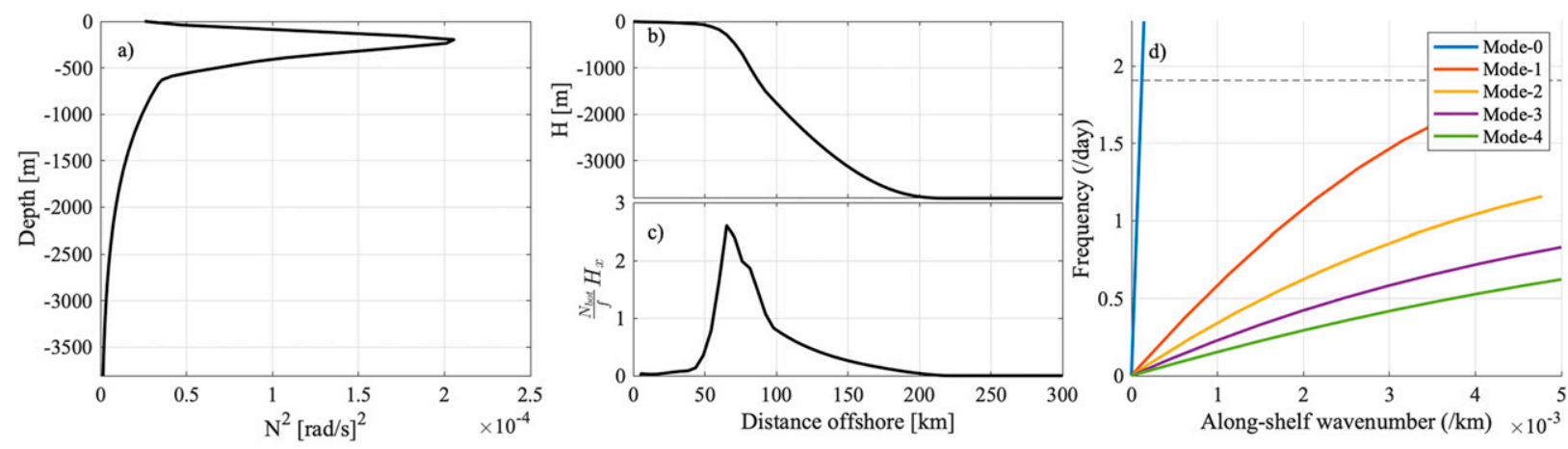

FIG. 1. (a) Stratification profile, (b) depth profile, (c) $\left(N_{\text {bot }} / f\right)(\partial H / \partial x)$, and (d) dispersion curves for the gravest (Kelvin wave) and first four coastal trapped wave modes. The horizontal dashed line indicates the inertial frequency.

and in a similar way for $\left\langle v_{2} p_{1}\right\rangle$. Thus, along-shelf flux has along-shelf structure in the flux pattens with beat periodicity (Crawford 1984). The total along-shelf flux is given by the cross-shelf and depth integral of Eq. (22), and for orthogonal modes the cross terms integrate to zero using Eq. (21) (see the appendix). For short waves, however, the cross terms are nonzero in the integral so that the total wave flux (and wave energy) is not simply the sum of individual wave fluxes (and energies) along the coast (Huthnance 1978).

\section{An example calculation of wave flux structure for a single mode}

The modal structure of stratified coastal trapped waves is strongly influenced by the stratification parameter

$$
S=\frac{N_{\max } L_{z}}{f L_{x}},
$$

which arises in the nondimensionalization of Eq. (6), where $L_{z}$ and $L_{x}$ are vertical and horizontal length scales, often taken to be the maximum shelf/slope depth and offshore extent. For very small values of $S$, the nodal lines of the modal structure in the offshorevertical plane become close to vertical, and the motion is nearly barotropic. For large values of $S$, nodal lines become close to horizontal, and the modal structure resembles a baroclinic Kelvin wave aligned along the sea bed (Wang and Mooers 1976). For intermediate values of $S$ the modal structure is neither strictly barotropic nor baroclinic.

In this section are illustrated the errors arising in calculating the wave flux associated with a trapped wave by assuming its energy to be strictly baroclinic, as would be the typical approach for freely propagating internal waves over flat bathymetry. Calculations are performed using the codes described in Brink (2006), which numerically solves linear Eq. (6) with the boundary conditions in Eqs. (7)-(10) on a sigma coordinate grid to determine the modal structures for a given stratification and cross-shelf topographic profile (Figs. 1a,b). Dispersion curves are computed using these codes to track resonant solutions in frequency-wavenumber space. The mode-1 structure determined from this linear model is additionally used to force a primitive equation numerical model [The Massachusetts Institute of Technology General Circulation Model (MITgcm), evolved from Marshall et al. (1997)] with identical bathymetry and stratification to the linear model (to the extent that the $z$-coordinate model allows). The model is run in hydrostatic mode with free slip boundary conditions at the partial cell topography (Adcroft et al. 1997) to be consistent with the linear solutions. In the forcing region, the along-shelf current is nudged toward the linear mode- 1 solution, which propagates out of the forced region along the shelf without change in structure, thus validating our linear solutions. Dispersion curves of the first five coastaltrapped waves show both the coastal Kelvin wave (mode 0 ), with largely barotropic structure, very fast phase speeds and large scales; and the higher internal modes, which are dispersive and have slower phase speeds (Fig. 1d). The parameter $H_{x} N_{\text {bot }} / f$ determines the nature of the dispersion curve: for values of $H_{x} N_{\text {bot }} / f>1$, the dispersion curves of each mode rise up to cross the inertial frequency, whereas for $H_{x} N_{\text {bot }} l f<1$ the curves reach a maximum frequency below the inertial period (Chapman 1983). For the stratification and topography in this example the modes cross the inertial period, becoming leaky costal trapped waves at superinertial frequencies (Figs. 1c,d).

The modal structures of the along- and offshore currents for modes $1-3$ with subinertial periods of around 2 days illustrate the mixed barotropic/baroclinic nature of the coastal trapped waves (Fig. 2). For this intermediate value of the stratification parameter $(S \approx 0.5)$, along-shelf currents have distinct vertical structures and are associated 


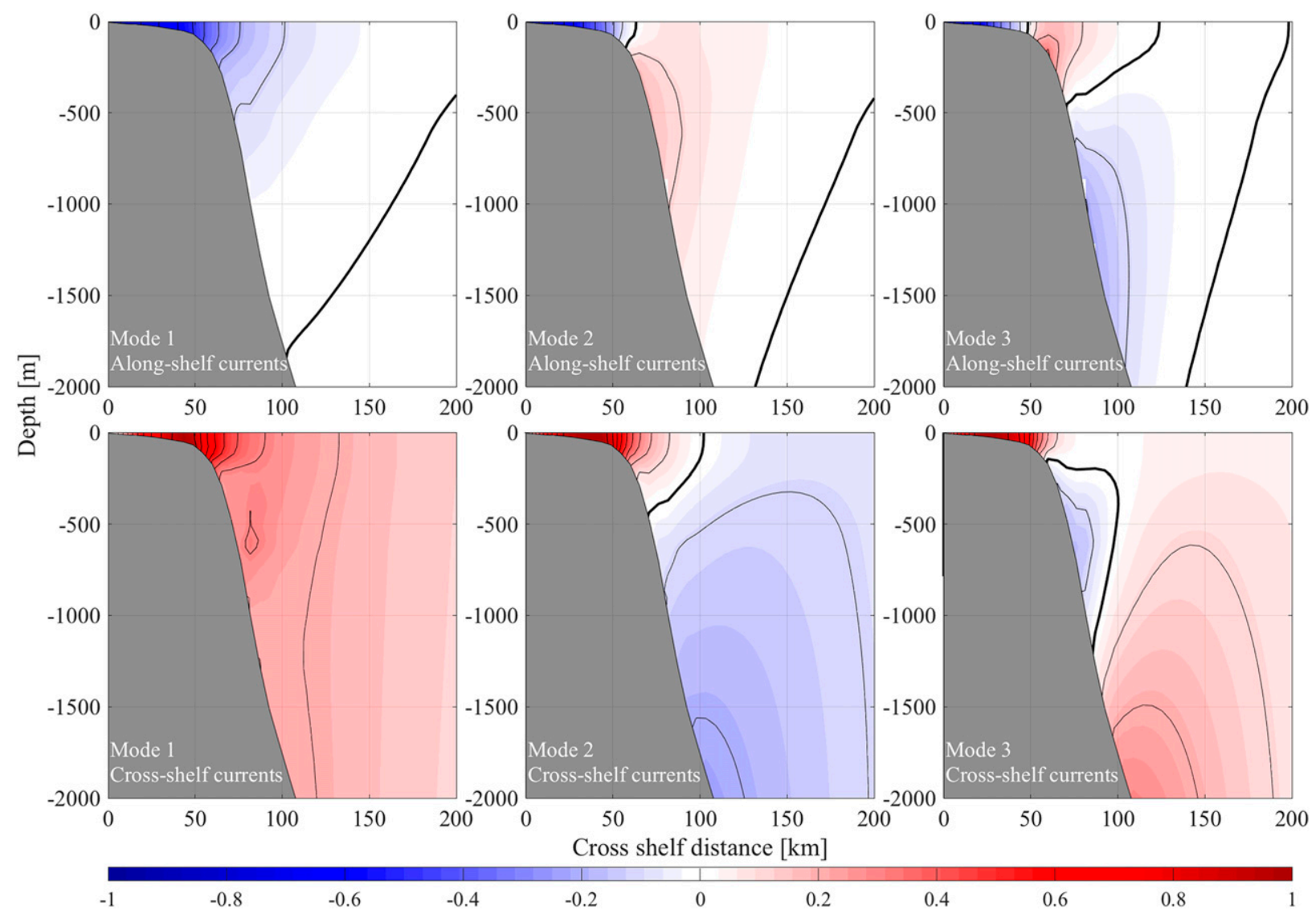

FIG. 2. Nondimensional velocity modal structures of modes 1-3 from the linear model, showing (top) along-shelf and (bottom) cross-shelf velocity components. Zero crossings are contoured with heavier weight.

with large depth mean currents. In this example, wave currents over the shelf tend to be much better approximated by a barotropic structure, while currents offshore are decidedly mixed. However, if the pycnocline were higher in the water column, or the shelf depth deeper, the structure on the shelf would become more baroclinic.

If the currents and pressure are decomposed into barotropic and baroclinic components as for freely propagating waves, the depth-integrated wave flux can be expressed as

$$
\langle\overline{v p}\rangle=\left\langle\overline{v_{\mathrm{bc}} p_{\mathrm{bc}}}\right\rangle+\left\langle\overline{\mathrm{v}_{\mathrm{bt}} p_{\mathrm{bt}}}\right\rangle
$$

where the subscripts "bc" and "bt" indicate baroclinic and barotropic, respectively, and the overbar signifies a depth average. The cross terms (e.g., $\left.\left\langle\overline{v_{\mathrm{bt}} p_{\mathrm{bc}}}\right\rangle\right)$ become zero for these vertical modes. Each of the terms in the above expression is calculated for the first three modes and is shown in Fig. 3. The flux computed from mode 1 in the MITgcm run (a fully nonlinear forward time integral) demonstrates good agreement with the linear calculations.
For all three modes the depth-integrated baroclinic flux has an offshore structure that is quite different from the structure of the wave flux, often having the opposite sign to the wave flux at any given off-shelf location, and having nonzero values only away from the shelf. The integrated baroclinic flux is a severe underestimate of the total wave flux, with the depth-offshore integral accounting for around $10 \%, 15 \%$, and $40 \%$ of the total wave flux for modes $1-3$ respectively.

\section{Summary}

While internal tides in the open ocean with weak topographic slopes are close to being purely baroclinic, internal tides over the coast are not. In general, all coastal trapped waves modes have structures that include both baroclinic and barotropic components, making it relatively difficult to separate the gravest from higher modes, especially over complex bathymetry. Assuming the coastal internal tide to be strictly baroclinic has significant consequences for the estimation of coastal wave flux, and the implications of such a 

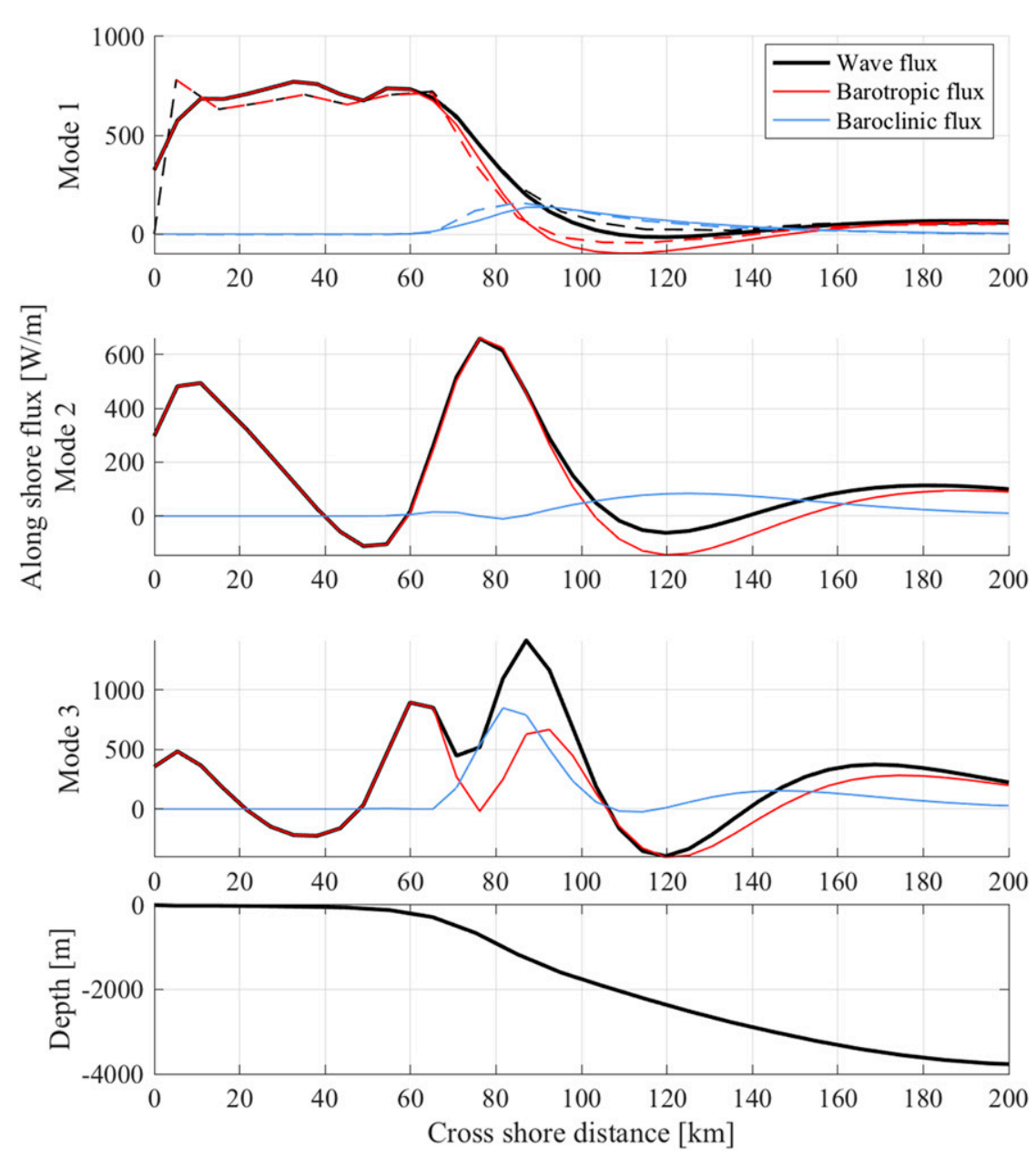

FIG. 3. Depth-integrated along-shelf wave fluxes calculated from linear model (solid lines) and primitive equation model (dashed lines, for mode 1 only). Wave flux for each mode is plotted in black, and the barotropic and baroclinic flux components are plotted in red and blue, respectively. Fluxes are scaled for a wave with along-shelf amplitude $v_{0}=0.1 \mathrm{~m} \mathrm{~s}^{-1}$.

decomposition computed in numerical models to date is that estimates of subinertial baroclinic tide flux at high latitudes are underestimates of the coastal trapped wave mode energy flux. The amount of this underestimate is sensitive to stratification and bathymetry, but in our example baroclinic flux accounts for only $10 \%$ of the wave flux in mode 1 . Though the conversion of energy between barotropic and baroclinic motions in the open ocean represents wave scattering from the gravest to higher wave modes, it must be interpreted with care at the coast as all coastal modes include barotropic and baroclinic components. In these regions, barotropic to baroclinic conversion simply identifies regions where scattering between modes changes the barotropic/baroclinic fractions of the propagating waves, and does not necessarily indicate a progression to higher modes. Because of the possibility of leaky coastal trapped waves at superinertial frequencies, it is reasonable to expect that this issue is not restricted only to high latitudes and that it applies in coastal regions even when the tides are superinertial.

Nevertheless, the baroclinic components of coastal trapped waves are still of interest for mixing as they are associated with vertical shears and isopycnal displacements that can lead to breaking, dissipation, and mixing within the water column. The conversion of barotropic to baroclinic energy, though not identifying any single wave scattering process, indicates a transformation of energy from motions that can dissipate in a bottom boundary layer, to motions that can dissipate high in the water column. As such, quantifying baroclinic energy flux and barotropic-baroclinic conversion can still be useful in coastal environments, but their interpretations are more limited than in the open ocean.

The properties of coastal trapped waves present challenges to tracking energy pathways near the coast 
via wave scattering as has been done in the open ocean (Carter et al. 2008). In the latter case, the energy flux carried by the internal tide is assessed far enough away from the conversion site, where the ocean bottom is almost flat and wave modes are well described by flat bottom modes. The energy lost from the barotropic tide is either dissipated at the conversion site (through processes such as hydraulic jumps and transient lee waves) or radiated away as internal tides, whose energetic properties can be quantified. In coastal areas, coastal trapped waves carry energy along the coast away from their generation site, however, an analogous analysis may be impossible to perform. Not only are there practical difficulties in identifying coastal trapped wave modes along a varying coast, the modes themselves are not, in general orthogonal, and the total wave flux is not a simple sum of individual wave modes away from the long wave limit. Observational difficulties are also increased: the two-dimensional modal structures of coastal trapped waves make it almost impossible to quantify the wave flux of a coastal trapped wave from a single point measurement, for example, a mooring, as the local energy flux for a single wave varies greatly in the offshore direction. Sufficient off-shelf data are required to either resolve the off-shelf structure of the flux, or at least allow a fit to the dominant modes that constitute the signal, from which the spatial integral of flux can be quantified.

Historically, tidal dissipation in shallow seas and coastal regions has been attributed to bottom friction (Taylor 1919; Jeffreys 1921; Egbert and Ray 2000). However, the presence of coastal trapped waves at both sub and superinertial frequencies raises the possibility that they may play an important role in setting the distribution of tidal dissipation and mixing in such locations, fluxing tidal energy along the coast and dissipating it where the coastal internal tide breaks. The same mechanism may also be relevant in the deep ocean away from coasts, where subinertial topographically trapped waves propagate along the ocean floor. Understanding the energetic properties of these bottom trapped waves may be important in building a global picture of dissipation and mixing in the ocean.

Acknowledgments. This work was supported by the Postdoctoral Scholar Program at the Woods Hole Oceanographic Institution, with funding provided by the Weston Howland Jr. Postdoctoral Scholarship, and by NSF under Grant OCE-1756781. I am grateful to K. Brink for the many useful conversations that contributed to this work and to J. Toole for providing detailed comments on an early version of this paper. The comments of three anonymous reviewers were very helpful in improving this paper.

\section{APPENDIX}

\section{Energy Flux Cross Terms in the Long Wave Limit}

To show that the energy flux for two wave modes $m$ and $n$ in the long wave limit is the sum of the flux of the individual waves, the cross terms must be zero [Eq. (22)]:

$$
\int_{-H}^{0} \int_{0}^{\infty}\left\langle v_{n} p_{m}\right\rangle+\left\langle v_{m} p_{n}\right\rangle d x d z=0 .
$$

To prove it, note that the alongshore flow is geostrophic so that $\left\langle v_{n} p_{m}\right\rangle \approx\left\langle\left(\partial_{x} p_{n}\right) p_{m}\right\rangle$ in the long wave limit and note that $\partial_{x}\left(\tilde{p}_{n} \tilde{p}_{m}\right)=\left(\partial_{x} \tilde{p}_{n}\right) \tilde{p}_{m}+\tilde{p}_{n}\left(\partial_{x} \tilde{p}_{m}\right)$. The integral can then be written in the form of the orthogonality relation:

$$
\begin{aligned}
\int_{0}^{\infty} \int_{-H}^{0} \frac{\partial}{\partial x}\left(\tilde{p}_{n} \tilde{p}_{m}\right) d z d x & =\int_{0}^{\infty}\left[\frac{d}{d x} \int_{-H}^{0} \tilde{p}_{n} \tilde{p}_{m} d z+\left.H_{x} \tilde{p}_{n} \tilde{p}_{m}\right|_{z=-H}\right] d x \\
& =\left.\int_{-H}^{0} \tilde{p}_{n} \tilde{p}_{m} d z\right|_{x=0}+\left.\int_{0}^{\infty} H_{x} \tilde{p}_{n} \tilde{p}_{m} d x\right|_{z=-H} \\
& =\delta_{n m} D_{n} f .
\end{aligned}
$$

\section{REFERENCES}

Adcroft, A., C. Hill, and J. Marshall, 1997: Representation of topography by shaved cells in a height coordinate ocean model. Mon. Wea. Rev., 125, 2293-2315, https://doi.org/10.1175/ 1520-0493(1997)125<2293:ROTBSC > 2.0.CO;2.

Brink, K. H., 1989: Energy conservation in coastal-trapped wave calculations. J. Phys. Oceanogr., 19, 1011-1016, https://doi.org/ 10.1175/1520-0485(1989)019<1011:ECICTW>2.0.CO;2.
- 2006: Coastal-trapped waves with finite bottom friction. Dyn. Atmos. Oceans, 41, 172-190, https://doi.org/10.1016/ j.dynatmoce.2006.05.001.

Carter, G. S., and Coauthors, 2008: Energetics of $M_{2}$ barotropicto-baroclinic tidal conversion at the Hawaiian Islands. J. Phys. Oceanogr., 38, 2205-2223, https://doi.org/10.1175/ 2008JPO3860.1.

Cartwright, D. E., 1969: Extraordinary tidal currents near St Kilda. Nature, 223, 928-932, https://doi.org/10.1038/223928a0. 
Chapman, D. C., 1983: On the influence of stratification and continental shelf and slope topography on the dispersion of subinertial coastally trapped waves. J. Phys. Oceanogr., 13, 1641-1652, https://doi.org/10.1175/1520-0485(1983)013<1641: OTIOSA $>2.0 . \mathrm{CO} ; 2$.

Clarke, A. J., 1977: Observational and numerical evidence for wind-forced coastal trapped long waves. J. Phys. Oceanogr., 7, 231-247, https://doi.org/10.1175/1520-0485(1977)007<0231: OANEFW $>2.0 . \mathrm{CO} ; 2$.

Crawford, W. R., 1984: Energy flux and generation of diurnal shelf waves along Vancouver Island. J. Phys. Oceanogr., 14, 1600-1607, https://doi.org/10.1175/1520-0485(1984)014<1600: EFAGOD $>2.0 . \mathrm{CO} ; 2$.

Dale, A. C., and T. J. Sherwin, 1996: The extension of baroclinic coastal-trapped wave theory to superinertial frequencies. J. Phys. Oceanogr., 26, 2305-2315, https://doi.org/10.1175/ 1520-0485(1996)026<2305:TEOBCT $>2.0 . \mathrm{CO} ; 2$.

Egbert, G., and R. D. Ray, 2000: Significant dissipation of tidal energy in the deep ocean inferred from satellite altimiter data. Nature, 405, 775-778, https://doi.org/10.1038/35015531.

Fer, I., M. Müller, and A. K. Peterson, 2015: Tidal forcing, energetics, and mixing near the Yermak Plateau. Ocean Sci., 11, 287-304, https://doi.org/10.5194/os-11-287-2015.

Hughes, K. G., and J. M. Klymak, 2019: Tidal conversion and dissipation at steep topography in a channel poleward of the critical latitude. J. Phys. Oceanogr., 49, 1269-1291, https:// doi.org/10.1175/JPO-D-18-0132.1.

Huthnance, J. M., 1978: On coastal trapped waves: Analysis and numerical calculation by inverse iteration. J. Phys. Oceanogr., 8, 74-92, https://doi.org/10.1175/1520-0485(1978)008<0074: OCTWAA $>2.0 . \mathrm{CO} ; 2$.

Jeffreys, H., 1921: Tidal friction in shallow seas. Philos. Trans. Roy. Soc. London, 221A, 239-264, https://doi.org/10.1098/rsta.1921.0008.

Kang, D., and O. B. Fringer, 2012: Energetics of barotropic and baroclinic tides in the Monterey Bay area. J. Phys. Oceanogr., 42, 272-290, https://doi.org/10.1175/JPO-D-11-039.1.

Kelly, S. M., 2016: The vertical mode decomposition of surface and internal tides in the presence of a free surface and arbitrary topography. J. Phys. Oceanogr., 46, 3777-3788, https://doi.org/ 10.1175/JPO-D-16-0131.1.

Klymak, J. M., H. L. Simmons, D. Braznikov, S. Kelly, J. A. MacKinnon, M. H. Alford, R. Pinkel, and J. D. Nash, 2016: Reflection of linear internal tides from realistic topography:
The Tasman continental slope. J. Phys. Oceanogr., 46, 33213337, https://doi.org/10.1175/JPO-D-16-0061.1.

Li, Z., J.-S. von Storch, and M. Müller, 2017: The $\mathrm{K}_{1}$ internal tide simulated by a $1 / 10^{\circ}$ OGCM. Ocean Modell., 113, 145-156, https://doi.org/10.1016/j.ocemod.2017.04.002.

Marshall, J., A. Adcroft, C. Hill, L. Perelman, and C. Heisey, 1997: A finite-volume, incompressible Navier Stokes model for studies of the ocean on parallel computers. J. Geophys. Res., 102, 5753-5766, https://doi.org/10.1029/96JC02775.

Masunaga, E., O. B. Fringer, Y. Kitade, H. Yamazaki, and S. M. Gallager, 2017: Dynamics and energetics of trapped diurnal internal Kelvin waves around a midlatitude island. J. Phys. Oceanogr., 47, 2479-2498, https://doi.org/10.1175/ JPO-D-16-0167.1.

Müller, M., 2013: On the space-and time-dependence of barotropic-tobaroclinic tidal energy conversion. Ocean Modell., 72, 242-252, https://doi.org/10.1016/j.ocemod.2013.09.007.

Musgrave, R. C., J. A. MacKinnon, R. Pinkel, A. F. Waterhouse, J. Nash, and S. M. Kelly, 2017: The influence of subinertial internal tides on near-topographic turbulence at the Mendocino Ridge: Observations and modeling. J. Phys. Oceanogr. 47, 2139-2154, https://doi.org/10.1175/JPO-D-16-0278.1.

Nash, J. D., M. H. Alford, and E. Kunze, 2005: On estimating internalwave energy fluxes in the ocean. J. Atmos. Oceanic Technol., 22, 1551-1570, https://doi.org/10.1175/JTECH1784.1.

Niwa, Y., and T. Hibiya, 2011: Estimation of baroclinic tide energy available for deep ocean mixing based on three-dimensional global numerical simulations. J. Oceanogr., 67, 493-502, https://doi.org/10.1007/s10872-011-0052-1.

Salmon, R., 1998: Lectures on Geophysical Fluid Dynamics. Oxford University Press, $400 \mathrm{pp}$.

Tanaka, Y., T. Hibiya, Y. Niwa, and N. Iwamae, 2010: Numerical study of $\mathrm{K}_{1}$ internal tides in the Kuril straits. J. Geophys. Res., 115, C09016, https://doi.org/10.1029/2009JC005903.

Tanaka, T., I. Yasuda, Y. Tanaka, and G. S. Carter, 2013: Numerical study on tidal mixing along the shelf break in the Green Belt in the southeastern Bering Sea. J. Geophys. Res. Oceans, 118, 65256542, https://doi.org/10.1002/2013JC009113.

Taylor, G. I., 1919: Tidal friction in the Irish Sea. Proc. Roy. Soc London, 96A, 330, https://doi.org/10.1098/rspa.1919.0059.

Wang, D.-P., and C. N. Mooers, 1976: Coastal-trapped waves in a continuously stratified ocean. J. Phys. Oceanogr., 6, 853-863, https://doi.org/10.1175/1520-0485(1976)006<0853: CTWIAC $>2.0 . \mathrm{CO} ; 2$. 